\title{
The Opportunities and the Limitations of using the Independent Post-Editor Technology in Translation Education
}

\author{
Burcu TÜRKMEN ${ }^{1}$, Muhammed Zahit $\mathrm{CAN}^{2}$ \\ Lecturer, Zonguldak Bulent Ecevit University, Applied English and Translation Programme, Zonguldak, TURKEY ${ }^{1}$ \\ $\mathrm{PhD}$. Student, Sakarya University, The Institute of Social Sciences, Translation Studies Department, Sakarya, TURKEY ${ }^{1}$ \\ Assistant Professor, Sakarya University, The Institute of Social Sciences Translation Studies Department, Sakarya, TURKEY ${ }^{2}$
}

\begin{abstract}
A new mechanical function known as post-editing, which helps to correct the imperfections of raw machine translation output, is introduced in the translation market. While this function is commonly used as an integral part of the machine translation, it can also be used for correcting non-translated texts on its own. The main purpose of this study is to find an answer to the question of which contributions could be made to the competences of translation students by using independent posteditors during academic translation education. A model course application and survey are conducted with the participation of students from translation studies departments. The interrater reliability is used in this study. In accordance with the results, it is found that most of the students have not known the usage of the independent post-editors during the translation process. The research provides new insights into the contributions of posteditor technology to the translation education. The findings of the research also reflect the contributions of post-editor technology to the translation quality in terms of the speed-time management, the accuracy of punctuations, abbreviations, and grammar rules. It is also determined that the post-editors contribute to the competencies of the translation students. As a result, it is suggested that the post-editors may be used as an educational material in translation education. Indeed, the results require other studies about the usage of post-editors as educational materials.
\end{abstract}

Keywords-Post-editors; machine translation; translation education; computer-assisted translation

\section{INTRODUCTION}

Using machine translation systems and the significance of the localization industry cannot be ignored in order to meet ever-increasing demands for translation services. Different translation service providers and customers are aware that using such systems is a viable solution for translation. Certain projects need to be completed within a short time and with a low budget. In this context, translation service providers' aim is to increase the translation efficiency, improve delivery of work and customer feedback times, and keep their work in a competitive environment.

According to TAUS [1], the quality of the translated texts received from the machine translation programs and the followed post-editing processes are at two different levels.

This research paper is an expanded and improved version of another research paper titled as Yabancı Dile Doğru Gerçekleştirilen Çevirilerde Bağımsız Son Biçimleyicilerin İşlevleri: Çeviribilim Öğrencileriyle Gerçekleştirilen Bir Uygulama Örneği in Turkish. Article Information: DOI: 10.18033/ijla.4026, January 2018, International Journal of Language Academy 6(25):45-61.
According to the first level of quality (limited post-editing), the text is called "good enough" if it can convey the meaning of the source text accurately and comprehensibly without making any grammatical or formal corrections. At this level, the editor must be sure that there is no mistaken addition or subtraction of the translation text in terms of its semantic correctness and in such a way that the meaning complexity in the target text can be created. The second level of quality (full post-editing), "publishable quality" means to be the same as expected from human translation. In addition to clarity and correctness, the translation output must be grammatically and formally correct. In this context, there are standards updated continuously by the International Organization for Standardization (ISO) [2].

Today's technology does not have a machine translation system that can be equal to the human translation output. Therefore, the quality of the translation obtained in the target language depends on human translators rather than machine translation. ISO, which is an international standards federation worldwide, working in partnership with a large number of state-owned and independent agencies and organizations (the International Organization for Standardization) published a standard Translation Services- Post-Editing of Machine Translation Output Requirements in 2017. This standard covers issues such as machine translation concepts, language and content creation concepts, the concepts about people and institutions, the concepts related to translation, the issues related to post-editing process, post-editing requirements of machine translation outputs, tasks of post-editors ${ }^{1}$. It can be said that these studies will continue and in this way, the interest on the post-editors will increase.

In this research, especially the independent post-editors are analysed. Under the literature review and discussion section, the studies and researches on post-editors integrated into machine translation and the systems of independent posteditors are introduced. Under the methodology section, research design, study group/the participants, the validity and the reliability of the research, procedures, data collection tools and data analysis steps are introduced. Then, the post-editors used in the research are introduced in a separate section.

\footnotetext{
${ }^{1}$ Full version of the standard is not open, information about the context is given. https://www.sis.se/api/document/preview/8027889/, accessed: 04.20.2018.
} 
Under the results and discussions section, the survey questions and their results received from 140 Translation Studies students from English and German minors, are evaluated and displayed. Under the self-control of students and evaluation of post-editors section, two different evaluations about posteditors are given. The evaluations are carried out by the students. The first evaluation is carried out before using the post-editors, the second one is carried out after using the posteditors while they were translating the chosen texts. Under the conclusion section, the contributions of the post-editors during the translation process are given and it is suggested to use post-editors during the translation education.

\section{LITERATURE REVIEW AND DISCUSSION}

In Translation Studies field, the neural machine translation has been seen as a revolution since its contributions to the high quality of the translated texts and saving the effort during the translation process. In this concern, post-editing systems offer a unique opportunity for improving neural machine translation systems [3]. Post-editing is also determined as the modification process of the machine translation output with a minimum labour effort rather than re-translation of the text. Automatic post-editing aims to correct the systematic and repetitive errors found in the output of machine translation [4]. In this concern, in another research, online automatic postediting system is introduced as trained on generic data and its exploiting user feedback to develop its performance. Online automatic post-editing system is evaluated on the output of neural machine translation systems. As indicated in the results of this research, from the view of resources and the technical expertise requirements, the current automatic post-editing technology in the typical setting of most Language Service Providers (LSPs) is seen effective [5].

According to García, the first recorded post-editor usage was blocked in the early 1960s. During the 1960s the posteditors were used by USA Airforce Foreign Technology Department and Euratom. However, when the financial support in the United States expired partially with a report prepared by Automatic Language Processes Committee (ALPAC) in 1966 if the post-editors are compared with human translation, was ended since it was thought that the post-editors are not worth the effort in terms of quality and usage difficulty. It is stated that during the 1970s, machine translation and post-editors were used by different European Union organizations and certain companies $[6]^{2}$.

In the research of Federico Gaspari and others, a survey was conducted with the participation of 438 stakeholders in the translation and localization sector and $30 \%$ of those reported using machine translation. While $38 \%$ of those using machine-translation indicated that their translation had already been through post-editing, $30 \%$ of them indicated that they did not use the post-editors at all, and $32 \%$ of the participants used post-editor at different levels [7]. The survey results indicate that using machine translation and post-editors in the direction of increasing demands is increasing day by day, and it is predicted that this will continue in the future [8], [9], [10]. ${ }^{3}$

\footnotetext{
${ }^{2}$ The post-editors are the ones integrated in machine translation.

${ }^{3}$ For further information read and compare.
}

Full version post-editors are often used in the texts that are more important and intended for publication on the market and are integrated with Computer Aided Translation (CAT) tools, translation memories, or dictionaries. On the other hand, limited post-editors are not often integrated with CAT tools. The limited post-editors which are generally available free of charge, may be used as the full version and fulfil more extensive functions when they are purchased. It is not a surprise that machine translation (MT) is often seen during business negotiations when the intensive pressures of localization services and the delivery of products to foreign markets are considered. It is known that a machine translation tool can translate faster. By using such tools, it is possible to translate thousands of words in a day in a cheaper way.

Since no human intervention is required in machine translation, it is the cost of these tools/programs only. However, when there is no human touch, the linguistic quality of the target text and the level of loyalty will be doubtful. The quality of the translation content received by using the machine varies and depends on many factors such as the quality and standardization level of the source material, the terminology suitability used to train the translation machine, the quality and size, the level of applied post-editing, the customer's low-quality definition and language pairs.

According to ISO 17100: 2015, post-editing means "editing and correcting the machine translation output" [11]. Allen noted the separation among the different levels of postediting. First, it describes the determinants of post-editor levels and suggests using translation input and output to categorize these levels. It is possible for machine translation in three different levels for translation output. These can be classified as 1) translation delivered without post-editing, 2) translation delivered with limited post-editing, and 3) translation delivered with full end-format [12]. Correspondingly to the ISO standard, the students translate a common text without using machine translation, then they use the independent post-editor programs on these translated texts, and the aforementioned limited and full post-edited texts are obtained [13].

Guerberof Arenas discusses the views and thoughts on post-editing of professional translators, especially those using machine translation in a competitive environment required by modern times, in a survey supported by a survey application. It is noted that the majority of professional translators participating in the survey are satisfied with the use of posteditors in terms of workflow and timing [14]. However, some of the translators expressed that they did not like to use posteditors. They stated the reasons for this as while getting a good machine translation, some machine translation programs have been experiencing problems such as low quality, the high number of instructions to complete small tasks, maintenance of terminology, the large number of reference materials and complicated matching. The disadvantages mentioned above vary according to the types of machine translation and posteditors used by the translators. Although post-editors have the disadvantages, professional translators consider that these programs will develop over time in the localization sector and have a considerable share of machine translation output and terminology qualities. 
According to another study, it is stated that the post-editing process is known as its contribution to the translation quality, and this quality extremely depends on automatic metrics of machine translation. Post-editor systems do not take the translator experiences into consideration, even though the translation processes of the professional and inexperienced translators are different. However, it is seen that, besides the punctuation, spell-check results and etc., the meaning shifts and the structural issues are shown to be good indicators of post-editing effort [15].

In this study, different from machine translation software, the post-editors were used by a specific group of students who were trained in English and German translation by giving information about the types and uses of independent posteditors, suggestions about the using post-editors. It is seen in the studies related to post-editors, they mainly analyse the programs integrated with machine translation. However, under this research, researches on independent programs (which are not integrated with machine translation) have been carried out. However, the students who use the post-editors express that they get positive results on their target texts and these opinions are supported with the evaluations of the survey data.

\section{RESEARCH QUESTIONS}

The research questions of the study are:

1) Whether the students who will work as translators/interpreters know the names of certain computerassisted translation tools, and the post-editors and their functions.

2) Whether the students (professional translator candidates) benefit from the post-editors by using them independent of the native languages- into German and English foreign languages.

3) Whether using such technologies while translating into a foreign language contributes to professional translator competences.

\section{METHODOLOGY}

\section{A. Research Design}

The purpose of the descriptive research is to describe a phenomenon and its characteristics [16]. Descriptive research includes certain types of research methods such as survey, correlation study, qualitative study, and content analysis. All this kind of types are different from the view of their data collection/analysis procedures, but not in data availability [17]. As it is seen, according to the descriptive research method, the current situation that is what has happened rather than how or why something has happened is concerned. Therefore, in order to collect data from the students and describe the results received from them, the survey has been conducted. The results are described and they indicate the current position of post-editors in translation education. The suggestion is given for improving the quality of translated texts into foreign languages and using post-editors as educational materials during translation education.

\section{B. Study Group/ Participants}

In the research, a survey has been applied to two different student groups from English and German translation departments to determine the knowledge and perceptions about post-editors. 52 students from Sakarya University [18] and 88 students from Zonguldak Bulent Ecevit University [19], totally 140 students, participated in this survey and the research voluntarily. It can be stated that the results obtained from this sample group can include other translation studies students who translate from Turkish, their native language, into German and English. By using a slightly weaker claim, it can also be advocated that the translators who translate from Turkish native language into German and English can be included to the research universe.

\section{The Validity and Reliability of the Research}

The qualitative research method is used in this research. Furthermore, with the purpose of indicating the validity and the reliability of the assessment, expert opinions are consulted. Common criteria are determined for the interrater reliability, and for the internal validity of the research, the triangulation technique (the consistency among the analysis of data from the survey, application/observation and self-evaluation) is followed. The interrater reliability is related to the kappa statistic. According to interrater reliability, the data is collected in the study and correct representations of the variables are measured. Measurement of the extent to which data collectors (raters) assign the same score to the same variable is called interrater reliability [20]. The questions of the survey are submitted to two Assistant Prof. Dr., as raters, in The Translation Studies Department at Sakarya University. In the simple correlation calculated between two raters, at a higher level and positive 0.97 relation is determined.

\section{Procedures}

The process for revealing the perceptions and applications of the students in a realistic and integrated approach is followed. In this research, translating from native languages into foreign languages is preferred because of the opinion of making more mistakes during the text production process in foreign language. The reason of preferring post-editors in foreign language is their potential for receiving an independent research result from native language, and in this framework, the international dimension increase of the research. Shortly after the translations are completed, a survey is prepared in order to find out whether the students use any program or tools for determining the reasons of faults while translating texts from Turkish into English or German, and to check the accuracy of the target texts. In the event that they use such programs and/or tools, it is aimed to learn what they are. In this concern, 7 survey questions are asked to the students.

\section{E. Data Collection Tools and Data Analysis}

Within the scope of qualitative research, the method of observation was collected via using the survey technique. The steps of the model application with post-editors are seen as below: 
Step 1: At first, a joint Turkish source text is required to be translated from Turkish native language into English and German languages.

Step 2: The translated texts are checked by using the posteditors.

Step 3: Finally, via a survey, the self-checking/ evaluation of the usability and the advantages of these programs before and after the usage of post-editors has been done.

Step 4: By the favour of the survey, views of students about their own translations before using the post-editors are received.

Step 5: Thus, at the second level, the students evaluate their own target texts after using the post-editors, and the differences occurred after the usage of post-editors in the target texts are determined. In the first application, the students are not informed about the post-editors, and it is controlled whether they know anything about these programs without having an education. At the end of the first application, right after the survey, students are informed about the post-editors and they are required to check their target texts via certain post-editors.

In the last part of the research, 4 questions are asked to the students (right after translating the joint text, they are required to evaluate their target texts by using post-editors). In the light of these questions, the students are required to

1) Score their target texts between 1-10 before using posteditors.

2) Score their target texts between 1-10 after using posteditors.

3) Answer whether the post-editors are beneficial in terms of translation quality.

4) Answer in what aspects the post-editors are beneficial.

Right after making the evaluations of the translated and checked texts by using the post-editors, it is seen that the aforementioned post-editors provide an improvement on the target texts. Through the data analysis of the survey results, the results of the spell-checker and the post-editors on the translations of joint texts, the final evaluations for quality and benefits of the post-editors and self-controls of students, the descriptive analysis are followed. The results of this descriptive analysis are correlated with the related studies in literature.

\section{POST-EDITORS USED IN THE RESEARCH}

In the context of the research, certain post-editors and spell-checkers are used for the translation practice and then for the supervision of the translated target texts of the students in English and German. The post-editor and spell-checker programs analyse the texts to find possible typos in the target texts obtained after the machine translation. These online programs offer more than 20 correction suggestions on their memories. In addition to the suggestions for corrections, thanks to the translation tabs in the same programs, machine translation databases such as Google Translate are also available. Some of these programs can also be used as a digital dictionary. Under this research, the online GoogleSEO, Duden and LanguageTool post-editors have been used in the translation control application carried out by German translators. During the translation control application carried out by Applied English and Translation Program students, they used the online LanguageTool and Reverso post-editors and the Spell Checker and Translate programs which can be downloaded to mobile phones. The information about how these programs work will be shared below. It is important for two reasons: firstly, it will be more transparent about how the research is conducted, and secondly, a general view of the working principles of the post-editors will be obtained.

\section{RESULTS AND DISCUSSION}

In this section, the data obtained from the survey and selfevaluation questions asked to the students who are studying Translation Studies, are described. Certain evaluations are combined and evaluated in the light of more than one data. Totally 140 Students, participating in the survey and translation application carried out within the scope of the current scientific research, continue their education in Bulent Ecevit University Applied English and Translation Program (88 students) and Sakarya University Department of Translation Studies- German (52 students). The students attend to the survey and translation practices on the basis of volunteerism.

\section{A. The Evaluations of Translation before using Post-Editors}

Question 1 of the survey is intended to determine the language pairs in which students' study. Question 2 is "Please indicate the reason for making mistakes in your translations from Turkish source language into English or German target language". In addition, more than one option offered by the response to the students. The reasons of making mistakes of students while they are translating are 1) to feel weakness in making preference words on target language, 2) to be unsure of the grammatical rules of the target language, 3) to be unsure of the writing rules on the target language, and 4) to be unsure about tense usages in the target language. Except for these reasons, they indicated the reasons for lack of attention and time problem. It is seen that the students have problems in preferring words while making translating texts from Turkish source language into English or German languages. It can be said that this results from not to know certain usages except for grammatical rules in the foreign languages, and not be sure about preferring the appropriate word.

In the question 3, the students are asked to answer "Which of the options do you prefer in order to create an accurate text in terms of spelling and grammatical rules, on a foreign language?". In addition, more than one option was offered by the response to the students. Under this question, it is desired to know what might be the reasons for creating sentences with missing points. It is aimed to receive the answers to this problem classified as auditory memory, visual memory and rule knowledge. $69.3 \%$ of the participant students answered that they remember the spelling of the words as a picture, that is, they use visual memories, $38.6 \%$ remember the grammatical rules and $37.1 \%$ answered that I remember the auditory spelling of the words. In the answers to this question, it is seen that most of the students stated that they remember words and uses thanks to their visual memories. 
In the question 4 , students are asked to rank the answers according to their priority order. The 4th question is "List the items which help to the right usage of spelling and grammatical rules in order to obtain a complete text in the foreign language". In order to facilitate classification of the answers, three reasons such as Auditory Memory, Visual Memory and Rule Knowledge are given to the students. For these three answers, the priorities are given separately. According to the answers, it is depicted that 78 people, $55.7 \%$ of all students, choose the Visual Memory option as the first priority among the items that help to the right usage of both spelling and grammatical rules in order to create complete text on the foreign language. In this context, it is understood that students keep the words in their minds as a picture. It can be concluded that the creation of complete text on foreign language is directly related to the visual memory of the students. It can also be thought that in the context of translation education, it is possible for the translation instructors to use the visual elements to help them develop their students' skills for the creation of complete texts in foreign languages.

The answers reveal that 38 people, $27.1 \%$ of all students, choose Auditory Memory as the first priority among the items which help to use both spelling and grammatical rules in order to create complete text on the foreign language. According to the answers, it is seen that 36 people, $25.7 \%$ of the students, choose Rule Knowledge as the first priority among the items that help to use both spelling and grammatical rules in order to create complete text on the foreign language. In this context, it is understood that students keep the words in their minds depending on their spelling and grammar rules. Questions 3 and 4 are directed to the students in an interconnected manner. In this context, it can be concluded that visual materials as learning material in translation education are more helpful to students. In contrast to the use of visual materials, it is also important that students who are studying foreign languages and translation have problems in pronunciation/phonetics. To make it clearer, Table I is set out below that summarizes the answers to questions 3 and 4 together.

TABLE I. GRAMMAR AND SPELLING ERROR PREVENTERS AND PRIORITIES

\begin{tabular}{|l|l|l|}
\hline & Quesiton 3: Preventer & Question 4: Priority \\
\hline visual memory & $69.3 \%$ & $55.7 \%$ \\
\hline rule knowledge & $38.6 \%$ & $25.7 \%$ \\
\hline auditory memory & $37.1 \%$ & $27.1 \%$ \\
\hline
\end{tabular}

For question 5, students are asked to rank the answers according to their priority order. Question 5 is "What do you use to check the correctness of your target texts (translations) while translating into foreign languages?". In this question, "four different options such as Parallel Texts, Computer Programs, The Person Whom I Trust His/Her Foreign Language and I Don't use Spell Checkers", are given to the students in order to facilitate the classification of the answers. For these four answers, the priorities are given in separately. According to the answers, parallel texts mean intending to control structures such as patterns and sentence structure through texts similar to translation texts completed into the source language and target language. According to the answers, the computer programs mean the spell-checking applications that are included in the Word Office Program and the spell-checkers in the machine translation programs such as Google Translate and/or Trados.

Here, the people who are trusted in their foreign language competences mean the teachers, teaching staff, sometimes family members or friends, who are considered to be at a higher level of knowledge of foreign language (English or German) than students. According to the answers, it is seen that only 6 of the 140 students who participated in the survey know and/or use the spell-checker programs. In this context, the majority of students are not aware of the spell-checkers. They frequently check the accuracy of the translations by using computer programs, then they search for parallel texts and consult with the teachers, teaching staff, successful friends, etc. to check their translations. It should be noted that the using of computer-aided supervision made by students will not be confused with the using of post-editors. When asked about the utilities that are used for translation, translation utilities such as Google Translate are expressed. This indicates that the learners are unaware of the post-editors. On the other hand, it is worth remembering that some translation machines or dictionaries, such as Google Translate, also provide spell checking and provide certain fixes, and in this respect, they can actually do spell-checking during the machine translation process.

In question 6, students are required to order their priorities "What kind of supporters did you use for the translation (English / German)?". Six different options to facilitate the classification of answers are offered in this question. These are, Online Dictionaries, searching on Google, Translation Software, Printed Dictionaries, People Whom I Trust on Their Foreign Language and Using Spell-Checker Programs. For these six answers, the priorities are given in separately. As it is seen from the answers, 124 people, $88.6 \%$ of the students, choose "I'm using Online Dictionaries" such as Google Translate, Tureng, Beluka, etc. as a 1st priority in response to the question. According to the answers, 24 people, $17.1 \%$ of the students, choose Searching on Google as the 1st priority in response to the 6th question, and 82 people, $58.6 \%$ of the students as the 2 nd priority. According to the data of this answer, most of the students, who have translation education, do not use translation software such as Trados, Google Toolkit and Wordfast or they use rarely. The obtained results indicate that most of the students do not give priority to using published (book) dictionaries, or they use rarely. The results reflect that students prefer using online dictionaries since their easy usages, being fast and facilitating to saving time. According to the data received from the question, it is seen that the students, even if rarely, students consult to the people whom they trust their foreign languages such as teachers, friends or family members, who are thought better educated or experienced than the students are.

It is seen that 8 people, $5.7 \%$ of the students, choose spellcheckers, and more than $70 \%$ of them do not use such programs or use rarely. According to the answers of this question, it is seen that the student check their translations in the target language on the basis of the meanings of the words, 
and they use the online translation programs, mainly the Google Translate application, to translate directly the sentences or phrases. The students state that while translating into the target language and then in the checking process of the translations, they search the texts as in sentences or by their titles from Google Search Engine since they think that comparing parallel texts and the similar structures may be useful. Below, the data is simplified and made clearer through Table II.

TABLE II. USED SUPPORTERS AS $1^{\text {ST }}$ AND $2^{\text {ND }}$ PRIORITY DURING THE TRANSLATION PROCESS

\begin{tabular}{|l|l|l|}
\hline & $\mathbf{1}^{\text {st }}$ priority & $\mathbf{2}^{\text {nd }}$ priority \\
\hline Online Dictionaries & $88.6 \%$ & $10.7 \%$ \\
\hline Searching on Google & $17.1 \%$ & $58.6 \%$ \\
\hline Using spell-checkers & $5.7 \%$ & $8.6 \%$ \\
\hline
\end{tabular}

Under the research, it is obtained that most of the students are unaware of the spell-checkers, which are important for the translation quality, and they have not used such kind of programs before. The 7 th question is prepared as in openended form. They are asked to answer the question "What are the translation utility software while translating from Turkish into foreign languages? How do you check your translations' correctness? The answers to this question are classified and evaluated. Within the context of this question, it is aimed to learn the translation utilities, software, programs such as online dictionaries, translation programs, websites and etc. which provide the usage opportunities in digital environments during the translation process. However, it is aimed to learn how the students check the correctness of their translations completed in the target language.

Within the context of this scientific research, two different student groups are available. The first group is from Applied English and Translation Program. It is seen that the aforementioned student group uses the online dictionaries such as Tureng, Sesli Sözlük, English-Turkish Dictionary, and Google Translate and Yandex Translate. However, they state that they use the parallel texts by searching from various search engines, consult to the people who have translation education and translation teaching staff and teachers from different forums in order to check the final forms of the translated texts.

On the other hand, the same question is asked to the second group which is from Translation Studies (German) Department. They stated that they use online dictionaries such as Tureng, Sesli Sözlük, Pons, Verbformen, Google.de, Cambridge, abkuerzungen.de, Beluka, canoonet, TDK, Glosbe, WordReference.com, bab.la, Langenscheidt OnlineWörterbuch, Konjugation.de, and Google Translate. Besides these, they stated that in order to check the accuracy of the translations, they search the parallel texts to find similar texts. They also consult with the people who have translation education and translation teaching staff from different forums in order to check the final forms of the translations. The answers of the students indicate that the methods, which students prefer for checking of the translations and the auxiliary tools during the translation process, corresponds the similar results. As a result of the first survey, it can be said that the students are not able to perform their own audits, they only use various online dictionaries and translation programs such as Google Translate among the auxiliary translation programs. However, it is clear that students don't check their texts' correctness for spelling and grammatical rules, and they don't use post-editors.

The students also stated that they check their translations by asking the people whom they trust their foreign language competences such as their teachers, translation lecturers or translators. Among the aims of this study, the importance of spell-checkers and post-editors in the translation education becomes clear. By using such programs during the translation education curriculums, the students can make their own audits on sentence structures, grammar and spelling rules, and can obtain more qualified texts in the target languages.

\section{B. The Self-Control of Students and Evaluation of Post- Editors}

The students in two groups (English and German) are asked to translate a joint text. During the text translation process, all students are free to use translation utilities, online dictionaries, etc. After collecting the first target texts, the survey above is applied to them and then, they are informed about the post-editors and they are asked to check their translations by using these post-editors.

The students are asked to evaluate themselves (their own translations) and the post-editors that they learn and use during the course with 4 questions. Firstly, the students are asked to "score their own translations between 1-10 before using post-editors (10 point means the best)". Students are asked to translate from Turkish source language to English or German target language. During the translation process, they are free to use every kind of online sources and applications that they know. The scores of students for their own evaluations are seen in a wide range between 3 and $10.10 \%$ of the students score between 3-4, 55\% scores between 5-7, and $35 \%$ scores between 8-10 point. According to this result, the average evaluation of their translation is 7 .

After completing the translations and self-evaluation, students are informed about post-editors and they are asked to check and edit their translations by using post-editors. Then, they are asked to "score their texts on which they use posteditors between 1 and 10". By completing the evaluation of the second target texts (before using post-editors), the students can see the differences between their first texts (after using post-editors).

Compared with the first evaluations, it is observed that there is a positive change. $8 \%$ of the students score 5-7, whereas $92 \%$ of the students score $8-10$. There is a significant difference between the translation of students that they don't use and use post-editors. In the light of this data, for the general evaluation of the translations, for the students, it can be said that the post-editors are satisfactory and give positive results. Table III will allow comparing the results more easily and highlighting the results. 
TABLE III. EVALUATION OF TARGET TEXT BEFORE AND AFTER USING POST-EDITORS

\begin{tabular}{|l|l|l|}
\hline & Percentage of Students & Score of Translations \\
\hline \multirow{2}{*}{$\begin{array}{l}\text { Before Using } \\
\text { Post-Editors }\end{array}$} & $55 \%$ & $5-7$ \\
\cline { 2 - 3 } & $35 \%$ & $8-10$ \\
\hline \multirow{2}{*}{$\begin{array}{l}\text { After Using } \\
\text { Post-Editors }\end{array}$} & $8 \%$ & $5-7$ \\
\cline { 2 - 3 } & $92 \%$ & $8-10$ \\
\hline
\end{tabular}

When the translation audits by using, post-editors are completed, the students participated in the research are asked what they think about the post-editors in respect of the translation quality in the target language.

For using post-editors, $0.7 \%$ of the students choose Neutral, $2.9 \%$ choose I think it is useless, 5\% choose I think it is a bit useful, quite a large group of student $91.4 \%$ choose I think it is useful (Table IV). It is seen that the data obtained from the previous question are parallel with the data of this question. While a general evaluation is asked in the previous question, more tangible data about the quality of the translations getting by using post-editors is tried to be obtained. According to this, it is seen that about $95 \%$ of the students agree that the post-editors make a positive contribution to the quality of the target text. It can be said that such data reveals the need for the post-editors to be analysed in terms of translation education.

TABLE IV. BENEFITS OF USING POST-EDITORS IN THE EYES OF THE STUDENTS

\begin{tabular}{|l|l|}
\hline Neutral & $0.7 \%$ \\
\hline I think it is useless & $2.9 \%$ \\
\hline I think it is a bit useful & $5 \%$ \\
\hline I think it is useful & $91.4 \%$ \\
\hline
\end{tabular}

Finally, the students are asked to answer the question of in which aspects do they think the post-editors are beneficial. The students are asked to order the benefits of post-editors in terms of their importance. As a result, they are agreed that the post-editors are beneficial: 113 students in the 1st line indicates the importance of translation quality, 2nd line indicates the speed-time for the delivery process of translation, 3rd line indicates the correct writing of the words, 4th line indicates the punctuation and spelling rules, 5th line indicates the grammatical rules, 6th line indicates the abbreviations, separate and compound writing rules. The 7 th line indicates the number of students who think that the post-editors should be in the translation education curriculums, and the 8th line indicates that post-editors make sense of being successful, competent and being independent of the outside of the translators during the translation process. According to the answers, it is seen that the post-editors are effective for providing the benefits above.

Based on the data received above, the advantages of the post-editors are determined. At this stage, the question to which extend the independent post-editors take place in the translation education curriculum, is asked. Under this research, the translation education curricula of 15 different European universities from 9 countries are analysed from their websites. It is seen that they include the translation technologies in their curricula, but especially any content about post-editors are not seen. This indicates that the translation studies departments in Europe neither give places in their curricula nor give details for post-editors.

\section{CONCLUSION}

Considering the survey and evaluations conducted under this research, it is determined that the independent post-editors are beneficial for the translation departments' students from the point of punctuation and spelling, grammar rules and have a positive effect on the quality of the translation into foreign languages. It is found that the post-editors are useful in terms of "speed-time" in order to contribute to the delivery process since they can quickly determine the mistakes of the text at the correction stage. However, it is seen that it helps with abbreviations, separate, contiguous writing rules, and the use of a high language instead of daily language. However, there is no way to check the correctness of the translation text in independent post-editors, a comparison with the source text is not possible. In this respect, it is also necessary to be careful about post-edited texts in terms of translation. Because each proposal does not guarantee that the original manuscript will remain true for its meaning.

As in the research, about the detailed evaluation of technological developments in terms of translators/ interpreters and the translation profession, carried out by Ersoy and Balkul, it is emphasized to integrate the contemporary technological changes and the new practices into the curricula of translation departments [21]. Therefore, translator trainees should not only prepare themselves for cultural and language-based conditions but also for future technologies and their advantages like using the independent post-editors as course materials during their educations. It is revealed under this research that the post-editors have different functions beyond the editing of the target text such as making sense of independence from the outside of the posteditors during the translation process. In this respect, making the post-editors a part of translation education should be emphasized in any native languages. Since the students are unaware of post-editors, it is necessary to integrate such programs into the courses related translation and technology such as Computer Assisted Translation. When the professional translator qualifications are considered, it can be said that translator candidates develop their technological, research and development competencies through the post-editors. It can be predicted that the translations can reach certain standards at this point. Literature review indicates that many researchers examine the post-editors integrated with the machine translation, whereas the post-editors can be used independently from the machine translation by the translators.

The research reveals that the post-editors are not known yet by the candidate translators. However, the translation application and the survey results indicate that the post-editors have important functions for completing texts in foreign languages. This necessitates another research about the posteditors being a translation education material. As a result of this study, it is indicated that using the post-editors during the 
translation process from any native language into English and German, as a foreign language, may give similar results.

Overall, it needs to be stressed that the findings of this research are limited to the evaluations of the practices and the questionnaires. However, further studies can illustrate the issue better. Certain research topics as given below could be the further research topics about the independent post-editors:

The research about the effects of the independent posteditors on translation quality: A research on different text types could be carried out and the performances of the independent post-editors on different types of texts, and the speed of the translators by translating with the post-editors could be studied.

The research about how could the independent post-editors be more functional in translation education and language education: A research about, beyond the post-editing function, how could the independent post-editors be more functional as education material if they had certain didactical purposes.

The research about how could the independent post-editors be more functional by using audible reading, attaching visuals and vocalising, adding sample sentences.

\section{REFERENCES}

[1] TAUS: (2016). TAUS Post-Editing Guidelines, Retrieved 21.04.2018, from https://www.taus.net/think-tank/articles/postedit-articles/taus-postediting-guidelines.

[2] KOPONEN, M. (2016), Is Machine Translation Post-editing Worth The Effort? A Survey of Research Into Post-editing and effort, The Journal of Specialised Translation, Issue 25- January 2016, University of Helsinki.

[3] PERIS, A.; CEBRIAN, L.; CASACUBERTA, F. (2017). Online Learning for Neural Machine Translation Post-Editing, Retrieved 25.12.2018, from https://www.groundai.com/project/online-learning-forneural-machine-translation-post-editing/.

[4] VU, T.T.; HAFFARI, G. (2018). Automatic Post-Editing of Machine Translation: A Neural Programmer-Interpreter Approach, Proceedings of the 2018 Conference on Empirical Methods in Natural Language Processing, p. 3048-3053. Brussels, Belgium, October 31-November 4 2018. 2018 Association for Computational Linguistics.

[5] NEGRI, M.; TURCHI, M.; BERTOLDI, N.; FEDERICO, M. (2018). Online Automatic Post-editing for Neural Machine Translation, Italian Conference on Computational Linguistics, Torino, Italy 10-11 December 2018. Vol. 2253, Retrieved from (25.12.2018) http://ceurws.org/Vol-2253/.

[6] GARCIA, I. (2012), "A Brief History of Postediting and of Research on Postediting." Anthony Pym and Alexandra Assis Rosa (eds) (2012) New Directions in Translation Studies. Special Issue of Anglo Saxonica 3(3), 292-310.

[7] GASPARI, F.; HALA, A.; DOHERTY, S. (2015). "A Survey of Machine Translation Competences: Insights for Translation Technology
Educators and Practitioners." Perspectives: Studies in Translatology, p. 1-26. Retrieved 25.05.2018, from http://dx.doi.org/10.1080/0907676X .2014.979842.

[8] DEPALMA, D. (2013). Post-editing in practice, Retrieved 21.04.2018, from http://www.tcworld.info/e-magazine/translation-and-localization/ article/post-editing-in-practice/.

[9] EDMUNDSON, H. P. and DAVID, G. H. (1958). "Research Methodology for Machine Translation.” Mechanical Translation 5(1), 815.

[10] GUERBEROF, A. A. (2010). "Project Management and Machine Translation." Multilingual 21(3), 1-4.

[11] ISO Translation services -- Post-editing of Machine Translation Output Requirements, Retrieved 05.04.2018, from https://www.iso.org/standard /62970.html.

[12] HU, K.; CADWELL, P. (2016). A Comparative Study of Post-editing Guidelines, Baltic J. Modern Computing, Vol. 4 (2016), No. 2, 346-353, Retrieved 21.04.2018, from https://pdfs.semanticscholar .org/ b01f/c3b465c02c0174262bbfbd67987fca1222be.pdf?_ga=2.151871418. 209486593.1527164074-584386589.1527164074.

[13] ALLEN, J. (2003). Post-editing. Computers and Translations: A Translator's Guide. 35, 297-317.

[14] GUERBEROF, A. (2009). Productivity and quality in MT post-editing, In Proceedings of MT Summit XII-Workshop: Beyond Translation Memories: New Tools for Translators MT, AMTA 2009 (26-30 Aug. 2009, Ottawa, Canada).

[15] DAEMS, J.; VANDEPITTE, S.; HARTSUIKER, R.J.; MACKEN, L. (2017). Identifying The Machine Translation Error Types with The Greatest Impact on Post-Editing Effort, Frontiers in Psychology, August 2017, Vol. 8, Article 1282.

[16] NASSAJI, H. (2015). Qualitative and Descriptive Research: Data Type Versus Data Analysis, Language Teaching Research, Vol. 19 (2) 129132.

[17] ATMOWARDOYO, H. (2018). Research Methods in TEFL Studies: Descriptive Research, Case Study, Error Analysis, R \& D, Journal of Language Teaching and Research, Vol.9 No.1, 197-204, January 2018.

[18] SAKARYA UNIVERSITY Department of Translation Studies, Retrieved 04.04.2018, from http://www.ceviribilim.sakarya.edu.tr/.

[19] BULENT ECEVIT UNIVERSITY Çaycuma Vocational High School Applied English and Translation Program, Retrieved 04.04.2018, from http://cmyo.beun.edu.tr/yabanci-diller/1/uygulamali-ingilizce-vecevirmenlik-programi.html.

[20] MCHUGH, M. L. (2012). Interrater Reliability: The Kappa Statistic, Retrieved 24.12.2018, from https://www.researchgate.net/ publication/232646799_Interrater_reliability_The_kappa_statistic

[21] ERSOY, H.; BALKUL, H. I. (2012). "Teknolojik Gelişmelerin Çevirmen ve Çeviri Mesleği Açısından Olumlu ve Olumsuz Etkileri: Çeviri Alanında Yeni Yaklaşımlar”, Akademik İncelemeler Dergisi (Journal of Academic Inquiries) Volume: 7, Number: 2, Year: 2012, Retrieved 15.12.2018, from https://www.academia.edu /3588396/ Teknolojik_Geli\%C5\%9Fmelerin_\%C3\%87evirmen_ve_\%C3\%87eviri _Mesle\%C4\%9Fi_A\%C3\%A7\%C4\%B1s\%C4\%B1ndan_Olumlu_ve_Ol umsuz_Etkileri_\%C3\%87eviri_Alan\%C4\%B1nda_Yeni_Yakla\%C5\%9 F\%C4\%B1mlar. 